\title{
Cardiovascular and metabolic abnormalities in the offspring of diabetic pregnancy
}

\author{
J. G. Manderson' 1 , B. Mullan², C. C. Patterson ${ }^{3}$, D. R. Hadden ${ }^{2}$, A. I. Traub ${ }^{1}$, D. R. McCance ${ }^{2}$ \\ ${ }^{1}$ Royal Jubilee Maternity Hospital, ${ }^{2}$ Regional Centre for Endocrinology and Diabetes, Royal Victoria Hospital, Belfast, UK \\ ${ }^{3}$ Department of Epidemiology and Public Health Medicine, Queen's University, Belfast
}

\section{Abstract}

Aims/hypothesis. Maternal fuel metabolism is known to exert long range effects on the later development of children of diabetic mothers. Recently cardiovascular disease in adult life has been linked retrospectively with foetal malnutrition. The aim of this study was to identify whether markers for fuel-related cardiovascular programming exist for the offspring of diabetic pregnancy.

Methods. Sixty-one children aged 5 to 11 years, of mothers with Type I (insulin-dependent) diabetes mellitus were compared with 57 randomly selected control children of non-diabetic mothers similar in age, sex and social class. Fasting blood was taken for plasma glucose, insulin, lipids, IGF-1, plasminogen activating inhibitor 1 (PAI-1) and the adhesion molecules ICAM-1, VCAM-1 and E-Selectin.

Results. Fasting glucose and insulin were similar in the two groups. Differences existed between the off- spring of diabetic and non-diabetic pregnancies (mean \pm SD) for total cholesterol $(4.45 \pm 0.56$ vs $4.18 \pm 0.66$, $p=0.03)$, LDL cholesterol $(2.73 \pm 0.49$ vs $2.39 \pm 0.54$, $p=0.001)$, Cholesterol-to-HDL ratio $(3.41 \pm 0.76$ vs $3.09 \pm 0.73, p=0.03)$, IGF-1 $(22.5 \pm 7.3$ vs $19.3 \pm 8$, $p=0.04)$, PAI-1 $(20.1 \pm 6.2$ vs $14.9 \pm 7.3, p=0.000)$, VCAM-1 $(1852 \pm 444$ vs $1509 \pm 385, p=0.000)$ and E-Selectin (geometric mean; 83.1 vs 63.9, $p=0.001$ ).

Conclusion/interpretation. These results indicate that important differences in cardiovascular risk factors exist between glucose-tolerant children of Type I diabetic and non-diabetic mothers, even in childhood. These data suggest that offspring of diabetic mothers might be at an increased risk for the development of vascular disease in later life. [Diabetologia (2002) 45:991-996]

Keywords Diabetic pregnancy, offspring of diabetic mothers, foetal programming, cardiovascular disease.
The proposal that maternal fuel metabolism might exert long range effects on human development was first suggested more than 40 years ago [1]. The hypothesis, with minor modification [2], is now widely accepted and envisages that excess foetal growth associated

Received: 3 December 2001 / Revised: 2 April 2002

Published online: 12 June 2002

(C) Springer-Verlag 2002

Corresponding author: Dr. D. R. McCance, Regional Centre for Endocrinology and Diabetes, Royal Victoria Hospital, Belfast, BT12 6BA, UK, E-mail: drmccance@hotmail.com Abbreviations: PAI-1, Plasminogen activating inhibitor 1; ICAM, intercellular adhesion molecule; VCAM, vascular adhesion molecule; HOMA-IR, homeostasis model assessment of insulin resistance; SDS, standard deviation score. with diabetic pregnancy is the consequence of foetal hyperinsulinism secondary to excess maternal glucose and other fuels. While subsequent data would generally support this concept, the relatively few studies which have examined the question are poorly comparable and focus almost exclusively on growth and glucose regulation [3, 4]. Confounding factors include variation in the number of subjects, year of birth, age at follow up, type of diabetes (pregestational, gestational), adequate control subjects and assessment of morbidities. During the intervening four decades, the management of pregnant women and their newborns has improved markedly.

Recently others have shown provocative epidemiological evidence that coronary heart disease, and several other chronic adult diseases have their origins 
in foetal and infant life [5]. It has been hypothesised that foetal undernutrition leads to a re-setting of the insulin axis, and possibly other hormones which control foetal growth, such as IGF-1, and adrenal steroids. These changes persist post delivery and lead to the metabolic features of the insulin resistance syndrome (glucose intolerance, hypertension, dyslipidaemia and central obesity). This hypothesis could simply represent a generalisation of the Pedersen-Freinkel paradigm, with such conceived fuel-related programming existing at both extremities of foetal nutrition [2].

Clustering of cardiovascular risk factors within individuals is now well recognised. Insulin resistance has been postulated to underpin these conditions [6]. The syndrome is considered to have genetic determinants [7]. Hyperactivity of the hypothalamic-pituitaryadrenal axis resulting from socio-economic and psychological stress in adult life might also be involved [8]. The data also raise the possibility that insulin resistance, hyperactivity of the HPA axis and the insulin resistance syndrome might have a common origin in foetal nutrition.

Plasminogen activator inhibitor-1 is the main inhibitor of the fibrinolytic system and increased concentrations have been found in patients with coronary artery disease [9]. Increased PAI-1 levels are recognised to be linked to other risk factors for vascular disease including hyperinsulinaemia, insulin resistance and urinary albumin excretion, at the time of diagnosis of Type II (non-insulin-dependent) diabetes mellitus [10]. This finding is not the result of obesity or coexistent atherosclerosis [10].

There is increasing evidence that atherosclerosis has an inflammatory pathogenesis [11]. Early in the formation of atherosclerotic lesions, leucocytes extravasate through the endothelium into the arterial wall. This process involves interactions between an array of cellular adhesion molecules, including the selectins (E-, P- and L-selectin) and members of the immunoglobulin superfamily (ICAM-1 and VCAM-1) [12]. Endothelial cell adhesion molecule over-expression might therefore be an early marker of atherogenesis [13].

The aim of our study was to examine whether foetal cardiovascular programming might result from foetal overnutrition by comparing metabolic and cardiovascular risk factors in the children of Type I diabetic women, aged 5-11 years, with a control group of children of non-diabetic women of similar age, sex and social class.

\section{Materials and methods}

Subjects. Type I diabetic pregnancies where the child was age 5 to 11 years were identified retrospectively from the maternity records of the Royal Jubilee Maternity Hospital in Belfast. They were compared with a group of offspring of non-diabetic mothers of similar age, sex and social class. Control subjects were selected randomly from a computer generated list of names. We recruited 80 mother and child pairs for each group. There were no significant differences in demographic characteristics between participants and non participants. Details of the index pregnancy were obtained from maternal records. All diabetic mothers were confirmed to have Type I diabetes before their pregnancy in question. Control mothers were excluded if they had a personal history or a first degree relative with any type of diabetes, if they had a medical history of a known metabolic disorder, or if they had had an abnormal OGTT in pregnancy. A routine screening programme for hyperglycaemia in pregnancy has been in use in this hospital for many years. Offspring of non-diabetic women were excluded if they were diabetic or had been investigated for diabetes. None of the offspring in either group had a diabetic father. Offspring were requested to fast for the assessment and were excluded if they did not comply. This left 61 offspring of diabetic mothers and 57 offspring of non-diabetic mothers for the study.

A standard questionnaire detailing medical history, family history of cardiovascular or metabolic disease and socio-economic status was obtained. Socio-economic status was assessed using the Standard Occupational Classification [14]. To simplify comparisons we divided subjects into manual labour and nonmanual labour groups. Ethical approval was obtained from the Ethical Committee of Queen's University, Belfast and after discussion with the parent and child, written consent was obtained from the parent or guardian of the participating children.

Clinical data. Measurements made on each child were, height (Leicester height measure, Child Growth Foundation to $1 \mathrm{~mm}$ accuracy), weight (Seca), triceps and subscapular skinfold thicknesses (Holtain skinfold caliper [15]). Pubertal status was assessed from an examination, and confirmed by the measurement of serum oestradiol and testosterone concentrations. Offspring were excluded from the results if serum oestradiol was more than $50 \mathrm{pmol} \cdot \mathrm{l}^{-1}$ or serum testosterone was more than $0.7 \mathrm{nmol} \cdot \mathrm{l}^{-1}$. After a five minute rest with the subject seated, systolic and diastolic blood pressures were measured using an automated device (Omron, HEM-711), with a cuff of the appropiate size for the measured mid-arm circumference.

Blood sampling. After the application of local anaesthetic cream (Lignocaine/Prilocaine, Emla cream, Astra), a blood sample was drawn from the antecubital vein for the measurement of plasma glucose, insulin, IGF-1, plasminogen activator inhibitor-1 (PAI-1), total cholesterol, LDL-cholesterol, HDLcholesterol, triglycerides and the adhesion molecules ICAM-1, VCAM-1 and E -Selectin. The samples were centrifuged within $4 \mathrm{~h}$ and the serum was stored at $-70^{\circ} \mathrm{C}$. Insulin resistance was calculated by HOMA, where $\mathrm{HOMA}=\left(\right.$ insulin, $\mathrm{mu} \cdot \mathrm{l}^{-1} \times$ glucose, mmol·1 $\left.{ }^{-1}\right) / 22.5$ [16].

Assays. Serum insulin was calculated using a commercially available microparticle enzyme immunoassay kit (Abbott laboratories, Tokyo, Japan ). Inter-assay and intra-assay coefficients of variation were less than $5 \%$ and the lower limit of detection was $1 \mu \mathrm{mol} \cdot \mathrm{ml}^{-1}$. IGF-1 concentration was assessed using a commercially available ELISA kit (Octeia IGF-1, Immunodiagnostic Systems, Boldon, UK). Inter-assay and intraassay coefficients were 6.95 to $7.14 \%$ and 2.3 to $3.5 \%$ respectively. The sensitivity of the assay was $0.25 \mathrm{nmol} \cdot \mathrm{l}^{-1}$. Serum oestradiol was measured using a commercially available radioimmunoassay (PerkinElmer Life Sciences, Wallac Oy, Turku, Finland). Inter-assay and intra-assay coefficients of variation were 3.6 to $8.3 \%$ and 3.9 to $5.2 \%$ respectively. The sensitivity of the assay was $0.05 \mathrm{nmol} \cdot \mathrm{l}^{-1}$. Serum testosterone was measured using a commercially available radioimmunoassay kit (Diagnostic Products, Los Angeles, Calif., USA). Inter-assay 
and intra-assay coefficients of variation were 4 to $15 \%$ and 5.9 to $11 \%$ respectively. The assay sensitivity was $0.14 \mathrm{nmol} \cdot \mathrm{dl}^{-1}$. PAI-1 was measured using a commercially available kit (Stachrom PAI, Diagnostica Stago, France). The lowest limit of detection is $5 \mathrm{mU} \cdot \mathrm{ml}^{-1}$. Soluble cell adhesion molecules (sICAM-1, sVCAM-1, sE-selectin) were measured using commercially available ELISA kits (Diaclone Research, Besancon, France). The intra-assay and inter-assay coefficients of variation were less than $6 \%$. Glucose, triglycerides and cholesterol were measured on an automatic Vitros 750 XRL analyser (Johnston and Johnston) by a colorimetric assessment at $540 \mathrm{~nm}$ wavelength. HDL analysis used a manganese and heparin precipitation in supernatant followed by standard cholesterol assay using a vitros analyser (Johnson and Johnson).

Statistical analyses. A study size of 63 offspring of diabetic mothers and 63 offspring of non-diabetic mothers was calculated to have $80 \%$ power to detect as statistically significant ( $p<0.05$; two-tailed) a difference in means between groups, as small as 0.5 of a standard deviation. To allow for anticipated levels of exclusion and non-response, 80 subjects in each group were invited to participate. All subjects attended for assessment, however a number were non-fasting and some also refused blood sampling. To allow for variations in birth weight by gestational age and sex, birth weight values were transformed to standard deviation scores (SDS) using the 1990 British Growth Standard [17]. The same approach was used to adjust BMI (weight/height ${ }^{2}$ ) for age at examination and sex. Quantitative variables are summarised using means and standard deviations, but those whose distributions were positively skewed were log transformed prior to analysis and are summarised using geometric means and interquartile ranges. Comparisons between diabetic and non-diabetic offspring are shown as differences in means (with $95 \%$ confidence limits) or as ratios of geometric means (with 95\% confidence limits) for log-transformed variables. Statistical significance was assessed using independent student's $t$ tests. Linear regression analysis was used to assess the association between cardiovascular and metabolic variables and birth weight SDS. All tests were conducted at the $5 \%$ level of significance.

\section{Results}

The sample size, sex of the children, mean age in years, weight, height, BMI, systolic and diastolic blood pressure, and triceps skin fold thickness did not differ between the groups (Table 1). The mean gestational age at delivery was lower in the offspring of diabetic mothers, compared with offspring of non diabetic mothers. Despite this, the distribution and the mean of the birth weights in the two groups were similar. Breast feeding rates at 6 weeks after delivery, smoking during pregnancy and social class were similar in both groups.

The mean plasma glucose, serum insulin and HOMA did not differ between the offspring of diabetic mothers and control offspring (Table 2). The mean serum IGF-1, total cholesterol and cholesterol-to-HDL ratio were statistically greater in the offspring of diabetic infants than in the control offspring. LDL cholesterol, PAI-1, VCAM-1, and E-Selectin were highly significantly greater in the offspring of diabetic infants than in the control offspring. HDL cholesterol and triglyceride concentrations were not statistically different between the two groups.

The metabolic and cardiovascular variables were examined within each group in relation to birthweight corrected for gestational age. None of the variables showed any significant associations in the offspring of control mothers. In contrast, in the offspring of diabetic mothers associations were observed; a one standard deviation increase in birthweight SDS was associated with a 0.22 increase in height SDS, a 0.20 increase in weight SDS, an $11 \%$ increase in triceps skinfold thickness, a $12 \%$ increase in insulin concentrations and a $1.23 \mathrm{mU} \cdot \mathrm{ml}^{-1}$ increase in PAI-1 (Fig. 1). Positive associations with birthweight SDS were also

Table 1. Clinical characteristics of offspring of diabetic and non-diabetic mothers

\begin{tabular}{lcc}
\hline & Offspring of diabetic mothers & Offspring of non-diabetic mothers \\
$n=61$ & $26 / 61(43 \%)$ & $28 / 57(49 \%)$ \\
\hline Male sex & $8.6(1.8)$ & $9.0(1.2)$ \\
Age (years) & $32.8(12.7)$ & $32.5(8.5)$ \\
Weight (kg) & $133.1(12.1)$ & $133.3(9.2)$ \\
Height (cm) & $18.0(3.9)$ & $18.0(3.1)$ \\
BMI (kg.m ${ }^{-2}$ ) & $0.59(1.35)$ & $0.60(1.21)$ \\
BMI SDS & $103.8(10.2)$ & $105.8(13.9)$ \\
Blood pressure systolic (mmHg) & $57.8(6.9)$ & $58.8(10.5)$ \\
Blood pressure diastolic (mmHg) & $14.2(6.2)$ & $13.2(5.5)$ \\
Triceps skin fold thickness (mm) & $37.0(2.0)$ & $39.4(1.8)$ \\
Gestational age at delivery (weeks) & $3410(716)$ & $3369(556)$ \\
Birth weight (g) & $1.41(1.56)$ & $0.01(0.90)$ \\
Birth weight SDS & $18 / 56(32 \%)$ & $23 / 54(43 \%)$ \\
Breast feeding & $13 / 57(23 \%)$ & $17 / 54(32 \%)$ \\
Maternal smoking & $24 / 61(39 \%)$ & $25 / 57(44 \%)$ \\
Manual occupation & & \\
\hline
\end{tabular}

${ }^{\text {a }} p<0.001$ for comparison of groups

SDS, standard deviation score

Data presented as mean (standard deviation) or number $(\%)$ as appropriate. 
Table 2. Comparisons of cardiovascular and metabolic variables between offspring of diabetic and non-diabetic mothers

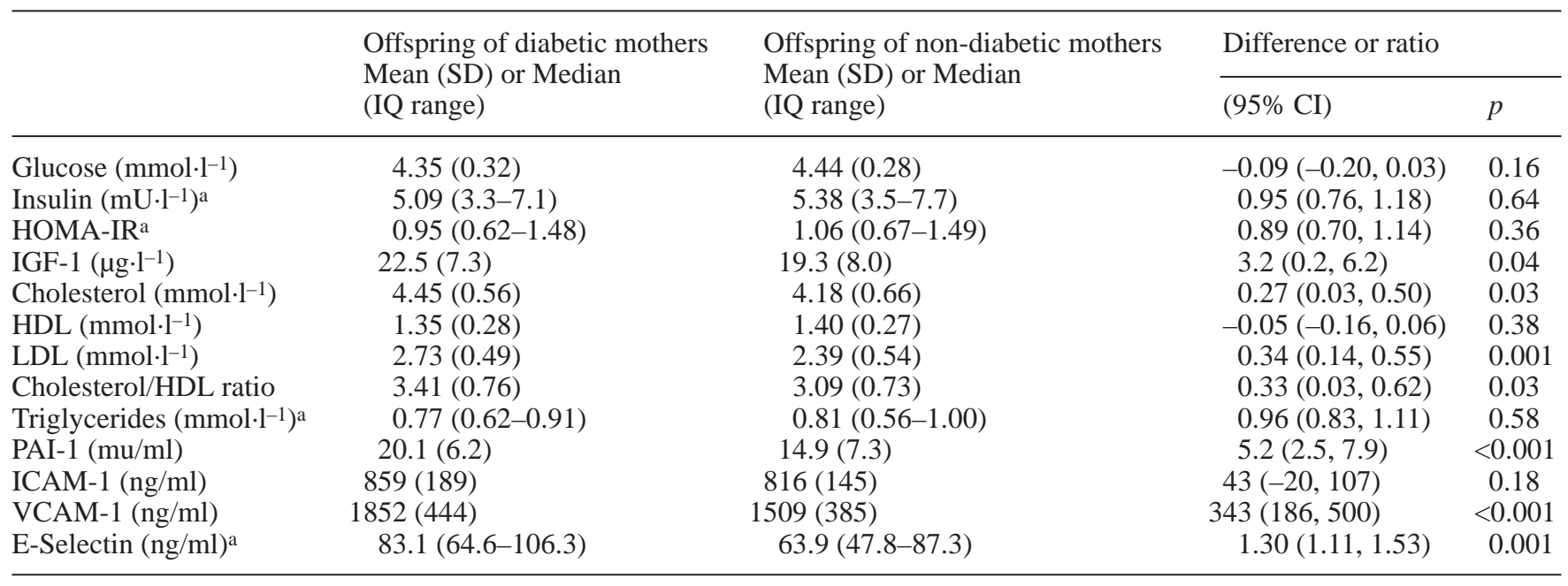

a Analysis carried out on logarithmically-transformed data

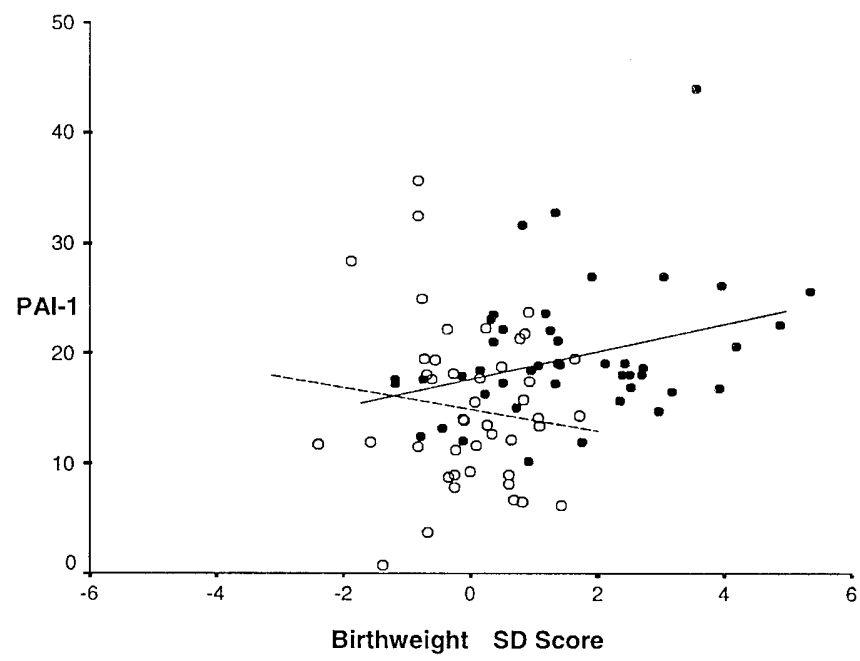

Fig. 1. Correlation between PAI-1 (mU/ML) and Birthweight SDS. Regression equations: Offspring of control mothers; PAI-1=15.3-1.0 Birthweight SDS ( $p=0.42)$. Offspring of Diabetic mothers; PAI-1=18.0+1.2 Birthweight SDS $(p=0.03)$

observed for HOMA ( $p=0.09)$, cholesterol-to-HDL ratio $(p=0.08)$, and E-selectin $(p=0.09)$.

For each variable that showed a difference between groups (Table 2), the mean in the diabetic offspring was adjusted to a mean birthweight SDS of 0.01 (the mean in the control offspring) using the fitted regression line. The differences between groups were generally reduced although the IGF-1 and cholesterolto-HDL ratio remained statistically significant.

Diabetic offspring were subdivided into those with a birthweight corrected for gestational age greater than the $90^{\text {th }}$ percentile $(49 \%)$ and those less than the $90^{\text {th }}$ percentile $(51 \%)$. Greater values were found in the greater than $90^{\text {th }}$ percentile compared with the less than $90^{\text {th }}$ percentile group for fasting insulin $(p=0.04)$ and triceps skinfold thickness $(p=0.001)$.

\section{Discussion}

Our study shows differences in metabolic, cardiovascular and inflammatory variables between offspring of Type I diabetic mothers compared with offspring of non-diabetic mothers at such a young age. The two groups did not differ in breast feeding rates at 6 weeks post delivery, which has been reported to be cardioprotective in some studies $[18,19]$, but not in others [20]. Maternal smoking during pregnancy was not different between the groups, nor was having a family history of cardiovascular disease.

Serum IGF-1 levels in offspring of diabetic mothers are higher than in the offspring of non-diabetic mothers. Others have shown that in low birthweight children aged 4 to 7 years, plasma IGF-1 concentrations are inversely related to birth weight and positively related to blood pressure in childhood after correction for current weight and height [21]. Our findings would suggest that there is a ' $U$ ' shaped association between IGF-1 and birthweight. Our data would also support the hypothesis that foetal programming might extend to hormones other than insulin. IGF-1 and insulin have also been implicated in the initiation of essential hypertension [22]. There are also several reports that low birth weight children have a higher $24 \mathrm{~h}$ urinary excretion of adrenal androgen and cortisol and cortisone metabolites [23]. In our study the increased IGF-1 concentrations in the offspring of diabetic mothers suggest that foetal over nutrition might have long-term implications in that these children could be at greater risk of developing hypertension as adults.

We found no statistically significant difference between the two groups for weight, height, BMI or triceps skinfold thickness. However, in the diabetic group, birthweight corrected for gestational age (birthweight SDS) was correlated with weight, height and triceps skinfold thickness. Several reports indicate that 
the offspring of diabetic women are taller [24, 25] than non-diabetic offspring but other studies report them as normal or short $[26,27,28]$. Reduced growth could result from cases of intra-uterine growth retardation caused by poor glycaemic control. Accelerated height is observed in children with obesity; however, obesity in childhood is not associated with increased adult height in the general population. Others [25] report a direct correlation between amniotic fluid insulin and relative obesity at the ages of 6 and 8 years suggesting a possible mechanism for the excess growth. Studies on Pima Indians living in Arizona who have a high prevalence of Type II diabetes, have shown that offspring aged 5 to 20 years (of women who had diabetes during pregnancy) were heavier in comparison to their height and more likely to be obese than the offspring of non-diabetic or prediabetic women (with NGT during pregnancy but in whom diabetes developed subsequently) [29].

In the diabetic offspring, birthweight corrected for gestational age also showed a correlation with fasting insulin and PAI-1. The association with insulin resistance, cholesterol-to-LDL ratio and E-Selectin approached statistical significance. These differences could not be explained solely by the increased birthweight for gestational age in the diabetic offspring. These findings indicate that maternal diabetes during pregnancy could lead to abnormalities of growth and insulin resistance in the offspring demonstrable even at a young age.

Those subjects born in the greater than $90^{\text {th }}$ percentile for birthweight corrected for gestational age had higher fasting insulin concentrations and larger triceps skinfold thickness. This suggests that larger babies (those more likely to be over-reaching their growth potential, i.e "macrosomic"), continue to have a higher amount of adiposity and insulin resistance as manifested by fasting insulin concentrations than those in the less than $90^{\text {th }}$ percentile group. This might predispose them to an increased risk of cardiovascular disease. It is not until we have a mechanism to show the underlying growth potential of each foetus that such data can be analysed fully.

Animal studies have shown that diabetes can be responsible for inducing cardiovascular dysfunction in adult offspring [30]. Few human studies have examined cardiovascular risk factors in offspring of diabetic pregnancy. Insulin resistance has been suggested as the primary pathology responsible for the increased cardiovascular risk. The lack of a difference between the two groups for systolic or diastolic blood pressure in our study could be explained by the relatively small sample size and the young age of the subjects. Pima Indians aged between 6 and 17 years of age showed an association between maternal diabetes with higher systolic but not diastolic blood pressure in the offspring adjusted for age, sex, relative weight and glucose and insulin concentrations [3]. In a cohort of
Type I and Type II diabetic patients from Chicago [4], offspring of diabetic mothers had higher systolic, diastolic and mean arterial pressures than a similar cohort of offspring of non-diabetic mothers [2]. Another study from the same centre showed higher systolic and mean arterial blood pressures, lower fasting low density lipoprotein concentrations, and lower total cholesterol in diabetic adolescent offspring compared with controls (31). We also found that there were quite marked lipid profile differences in the offspring of diabetic subjects with increases in the total cholesterol ( $p=0.03)$, cholesterol-to-HDL ratio $(p=0.03)$ and LDL cholesterol $(p=0.001)$.

Increased plasma concentrations of soluble adhesion molecules have been shown to predict cardiovascular disease in healthy men [32]. If cardiovascular disease does have a foetal origin, then endothelial cell adhesion molecule up-regulation should also begin early in life. We found considerable differences in these vascular markers of inflammation between the offspring of diabetic and control subjects. All the soluble cell adhesion molecules were increased in the diabetic offspring and significantly so for sVCAM-1 and sE-Selectin. A recent paper reported higher sICAM-1 concentrations in children with a strong family history of cardiovascular disease compared with those without a family history of cardiovascular disease [33]. This study suggested that children with a genetic predisposition for atherosclerosis have early evidence of endothelial dysfunction and adhesion molecule up-regulation. Our data suggest that inflammation could also be programmed by an adverse intrauterine environment.

Our results indicate that glucose tolerant children of Type I diabetic mothers have cardiovascular and metabolic abnormalities compared with a similar group of children of non-diabetic parents. These findings suggest that foetal overnutrition could be associated with the development of cardiovascular disease in later life.

Acknowledgements. D. McCance and J. Manderson proposed the initial study and secured the funding. J. Manderson and B. Mullan carried out the assessments. All authors contributed to the study design, data analysis and writing. D. McCance supervised the project. The study was supported by grants from The Department of Health and Social Services, Northern Ireland; Northern Ireland Mother and Baby appeal; The Metabolic Unit Research Fund, Royal Victoria Hospital, Belfast; The Royal Maternity Hospital, Royal Victoria Hospital, Belfast. Professor I. Young of the Department of Medicine, Queen's University, Belfast, provided invaluable help in measuring the adhesion molecules.

\section{References}

1. Pedersen J (1977) The pregnant diabetic and her newborn: problems and management. Williams \& Wilkins, Baltimore

2. Freinkel N (1980) Banting Lecture 1980: Of pregnancy and progeny. Diabetes 29:1023-1035 
3. Silverman BL, Purdy L, Metzger BE (1996) The intrauterine enviroment: implications for the offspring of diabetic mothers. Diabetes Rev 4:21-35

4. Pettitt DJ, Bennett PH (1995) In: Diabetes Mellitus in Pregnancy. Churchill Livingstone, New York, pp 379388

5. Barker DJP, Winter PD, Osmond C, Simmonds SJ (1993) The relation of head size and thinness at birth to death from cardiovascular disease in later life. BMJ 306:422-426

6. Reaven GM (1988) Banting lecture 1988; role of insulin resistance in human disease. Diabetes 37:1595-1607

7. McCance DR, Pettitt DJ, Hanson RL, Jacobsson LTH, Knowler WC, Bennett PH (1994) Birth weight and non insulin dependant diabetes: thrifty genotype, thrifty phenotype, or surviving small baby genotype? BMJ 308:942-945

8. Rosmond R, Dallman MF, Bjorntorp P (1998) Stress related cortisol secretion in men: relationships with abdominal obesity and endocrine, metabolic and haemodynamic abnormalities. J Clin Endocrinol Metab 83:1853-1859

9. Hamsten A, Defaire U, Waldius G et al. (1987) Plasminogen activator inhibitor in plasma: risk factor for recurrent myocardial infarction. Lancet 2:3-9

10. Gough SCL, Rice PJS, McCormack L, Chapman C, Grant PJ (1993) The relationship between plasminogen activator inhibitor-1 and insulin resistance in newly diagnosed type 2 diabetes mellitus. Diabet Med 10:638-642

11. Ross R (1999) Atherosclerosis - an inflammatory disease. New Engl J Med 340:115-126

12. Malik IS, Haskard DO (1999) Soluble adhesion molecules in ischaemic heart disease. Eur Heart J 20:990-991

13. Murray P, Frampton G, Nelson PN (1999) Cell adhesion molecules. BMJ 319:332-334

14. Office of Population Censuses and Surveys 1991 Standard Occupational Classification, vol 3. Social classifications and coding methodology. HMSO

15. Tanner JM (1962) The measurement of body fat in man. BMJ 1:187-196

16. Matthews DR, Hosker JP, Rudenski AS, Naylor BA, Treacher DF, Turner RC (1985) Homeostasis model assessment: insulin resistance and beta-cell fasting plasma glucose and insulin concentrations in man. Diabetalogia 28:412-419

17. Cole TJ, Freeman JV, Preece MA (1998) British 1990 growth reference centiles for weight, height, body mass index and head circumference fitted by maximum penalised likelihood. Stat Med 17:407-429

18. Singhal A, Cole TJ, Lucas A (2001) Early nutrition in preterm infants and later blood pressure: two randomised trials. Lancet 357:406-407
19. Ravelli AC, Meulen JH van der, Osmond C, Barker DJ, Bleker OP (2000) Infant feeding and adult glucose tolerance, lipid profile, blood pressure. Arch Dis Child 82:248252

20. Leeson CP, Kattenhorn M, Deanfield JE, Lucas A (2001) Duration of breast feeding and arterial distensibility in early pregnancy. BMJ 322:625-626

21. Fall CHD, Pandit AN, Law CM et al. (1995) Size at birth and insulin-like growth factor- 1 concentrations. Arch Dis Child 73:287-293

22. Lever AF, Harrap SB (1992) Essential hypertension: a disorder of growth with origins in childhood? J Hypertens 10:101-120

23. Clark PM, Hindmarsh PC, Shiell AW, Law CM, Honour JW, Barker DJ (1996) Size at birth and adrenocortical function in childhood. Clin Endocrinol (Oxf) 45:721-726

24. White P (1960) Childhood diabetes: its course, and influence on the second and third generations. Diabetes 9:345355

25. Silverman BL, Rizzo T, Green OC et al. (1991) Long-term prospective evaluation of offspring of diabetic mothers. Diabetes 40 [Suppl 2]:121-125

26. Farquhar JW (1969) The child of the diabetic woman. Arch Dis Child 44:36-47

27. Hagbard IO, Olow I, Reinand T (1959) A follow up study of 514 children of diabetic mothers. Acta Paediatr Scand 48:184-197

28. Breidahl HD (1966) The growth and development of children born to mothers with diabetes. Med J Aust 1:268-270

29. Pettitt DJ, Baird HR, Aleck KA, Bennett PH, Knowler WC (1983) Excessive obesity in offspring of Pima Indian women with diabetes during pregnancy. $\mathrm{N}$ Engl J Med 308:242-245

30. Holemans K, Gerber RT, Meurrens K, De Clerck F, Poston L, Van Assche FA (1999) Streptozotocin diabetes in the pregnant rat induces cardiovascular dysfunction in adult offspring. Diabetalogia 42:81-89

31. Cho NH, Silvermann BL, Rizzo TA, Metzger BE (2000) Correlations between the intrauterine metabolic enviroment and blood pressure in adolescent offspring of diabetic mothers. Paediatr 136:587-592

32. Ridker PM, Hennekens CH, Roitman-Johnson B, Stampfer MJ, Allen J (1998) Plasma concentrations of soluble intercellular adhesion molecule-1 and risks of future myocardial infarction in apparently healthy men. Lancet 351:88-92

33. Wojakowski W, Gminski J (2000) Soluble ICAM-1, VCAM-1 and E-selectin in children from families with high risk of atherosclerosis. Int J Mol Med 7:181-185 